\title{
Rapid detection of influenza virus subtypes based on an integrated centrifugal disc
}

Yuhan Yao $^{1}$, Xi Chen ${ }^{1}$, Xinlian Zhang ${ }^{1}$, Qi Liu ${ }^{1}$, Jinhui Zhu ${ }^{1}$, Wang Zhao ${ }^{1}$, Sixiu Liu ${ }^{1}$ and Guodong Sui ${ }^{*}, 1,2$

*To whom correspondence shall be addressed.

${ }^{1}$ Shanghai Key Laboratory of Atmospheric Particle Pollution and Prevention (LAP ${ }^{3}$ ), Department of Environmental Science \& Engineering, Fudan University, 220 Handan Road, Shanghai, 200433, P. R. China.

${ }^{2}$ Jiangsu Collaborative Innovation Center of Atmospheric Environment and Equipment Technology (CICAEET), Nanjing University of Information Science \& Technology, Nanjing, 210044, P. R. China 
Table S1 The primer sequences used in the article.

\begin{tabular}{|c|c|c|}
\hline $\begin{array}{l}\text { Assay } \\
\text { type }\end{array}$ & $\begin{array}{l}\text { Primer } \\
\text { name }\end{array}$ & Sequence (5' to 3 ') \\
\hline \multirow[t]{23}{*}{$\begin{array}{l}\text { RT- } \\
\text { LAMP }\end{array}$} & H1-F3 & AAGCTCAGCAAATCCTACA \\
\hline & H1-B3 & TCCCTCACTTTGGGTCTT \\
\hline & H1-FIP & GACTTTGTTGGTCAGCACTAGTAGTAGATTTTAAAGGGAAAGAAGTCCTCG \\
\hline & H1-BIP & ATCAGAATGCAGATGCATATGTTTTGCTATTTCCGGCTTGAACT \\
\hline & H1-LF & CGATACCCCGTAAGTGGTAG \\
\hline & H1-LB & TTTTGTGGGGACATCAAGATACAG \\
\hline & H3-F3 & AGGATGGGGGCTGTAACC \\
\hline & H3-B3 & CCAGCCATTTGCTCCATAGC \\
\hline & H3-FIP & TGAGACCTGTGCTGGGAGTCAGGTGGCATTTGGCCTGGTA \\
\hline & H3-BIP & TAGGCAGATGGTGGCAACAACCTGTAGTGCTGGCCAAAACC \\
\hline & H3-LF & AATCTGCTCACATGTTGCACA \\
\hline & H3-LB & CATTAATAAAACATGAGAACAGAAT \\
\hline & H5-F3 & ATTCCACAACATACACCC \\
\hline & H5-B3 & CTATGGTGATACCCATA \\
\hline & H5-FIP & TTTCTGAGTCCAGTCGCAAGGACTACACCATCGGGGAGTGC \\
\hline & H5-BIP & AAAAGAGAGGACTATTTGGAGCAACCATCTACCATTCCC \\
\hline & H5-LF & TATAGCAGGTTTTATAGAGGGA \\
\hline & H5-LB & CTGTTTGATTTCACATATTTGGG \\
\hline & H7-F3 & CGGAACCAAAGTAAACACATT \\
\hline & H7-B3 & ССТСТСАATAATTAAATCGGCTG \\
\hline & H7-FIP & GAGCAGATCCTGGGGATGTTTGCTGAAAGAGGAGTGGAAGTC \\
\hline & H7-BIP & AAGGACAGTTGACCTCGGTCAAAATTCTAGGAATTGGTCACA \\
\hline & H7-LF & CCACTGTTTCAGTTGCATTGAC \\
\hline
\end{tabular}


H7-LB ATGTGGACTCCTGGGGACA

H9-F3 GGAAAGGATGTTTCGAGCT

H9-B3 ATCACAAGATGAGGCG

H9-FIP ACTTCCTCCTGTTGTAGGTCCTTTTCCACAAATGTGATGACCAGTG

H9-BIP GACAGAAAATAGAGGGGGTCAAGCTTTTCAGTCGAATAAATGGTGAGG

H9-LF CATTCCGAATTGTCTCCATG

H9-LB TGGAGTCTGAAGGAACTTAC

FluB-F3 AGGGACATGAACAACAAAGA

FluB-B3 CAAGTTTAGCAACAAGCCT

FluB-FIP TCAGGGACAATACATTACGCATATCGATAAAGGAGGAAGTAAACACTCA

FluB-BIP TAAACGGAACATTCCTCAAACACCACTCTGGTCATAGGCATTC

FluB-LF TCAAACGGAACTTCCCTTCTTTC

FluB-LB GGATACAAGTCCTTATCAACTCTGC

RT-

PCR H1-F

H1-R ATACAACTTGTCAAACACC

H3-F TCTTGCCTGCCTGCTTGT

H3-R CTGCGGATGCCTTCTGTT

H5-F AATGCACAAGGAGAGGGAACTGC

H5-R TGATGCCCCGAAGCTAAACCA

H7-F CCTGGTATTCGCTCTGATTGC

H7-R GCACCGCATGTTTCCATTCT

H9-F CTCCACACAGAGCACAAT

H9-R GTTGTCACACTTGTTGTTGT

FluB-F CGCGGATCCGCCACCATGAAGGCAATAATTGTACTACTCATG

FluB-R CCGGAATTCTCAATAACGTTTCTTTGTAATGATGAC

Table S2 Regents in the LAMP system. 


\begin{tabular}{ll}
\hline LAMP mixture $(\mathbf{2 5} \boldsymbol{\mu L})$ & Concentration \\
\hline Tris-HCl (pH 8.8) & $20 \mathrm{mM}$ \\
$\mathrm{KCl}$ & $10 \mathrm{mM}$ \\
$(\mathrm{NH} 4) 2 \mathrm{SO} 4$ & $10 \mathrm{mM}$ \\
Triton X-100 & $1 \%$ \\
Each deoxynucleoside triphosphate (dNTP) & $1.4 \mathrm{mM}$ \\
Fluorescent detection reagent (calcein/SYBR Grren I) & $0.5 \mu \mathrm{L}$ \\
Bst DNA polymerase & $8 \mathrm{U}$ \\
Inner primers (FIP and BIP) & $1.6 \mathrm{M}$ \\
Outer primers (F3 and B3) & $0.2 \mathrm{M}$ \\
Loop primers (LF and LB) & $0.6 \mathrm{M}$ \\
Template & $5 \mu \mathrm{L}$ \\
\hline
\end{tabular}
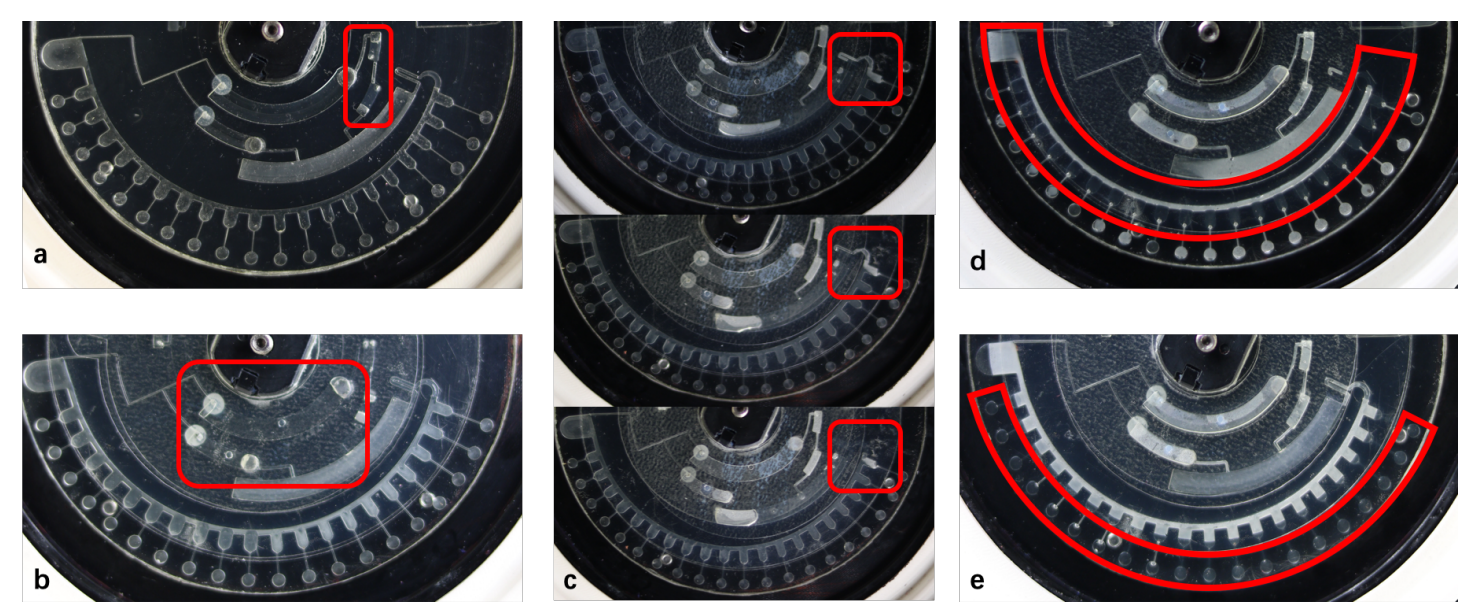

Figure S1 Photographs of disc work process. Two kind of enzymes (a), reaction buffer and sample (b) were loaded in the chambers. The regent flowed into next channel via siphon channel slowly because of siphonage (c). Reaction regent in the aliquoting structure (d) and RT-LAMP reaction chambers (e). 

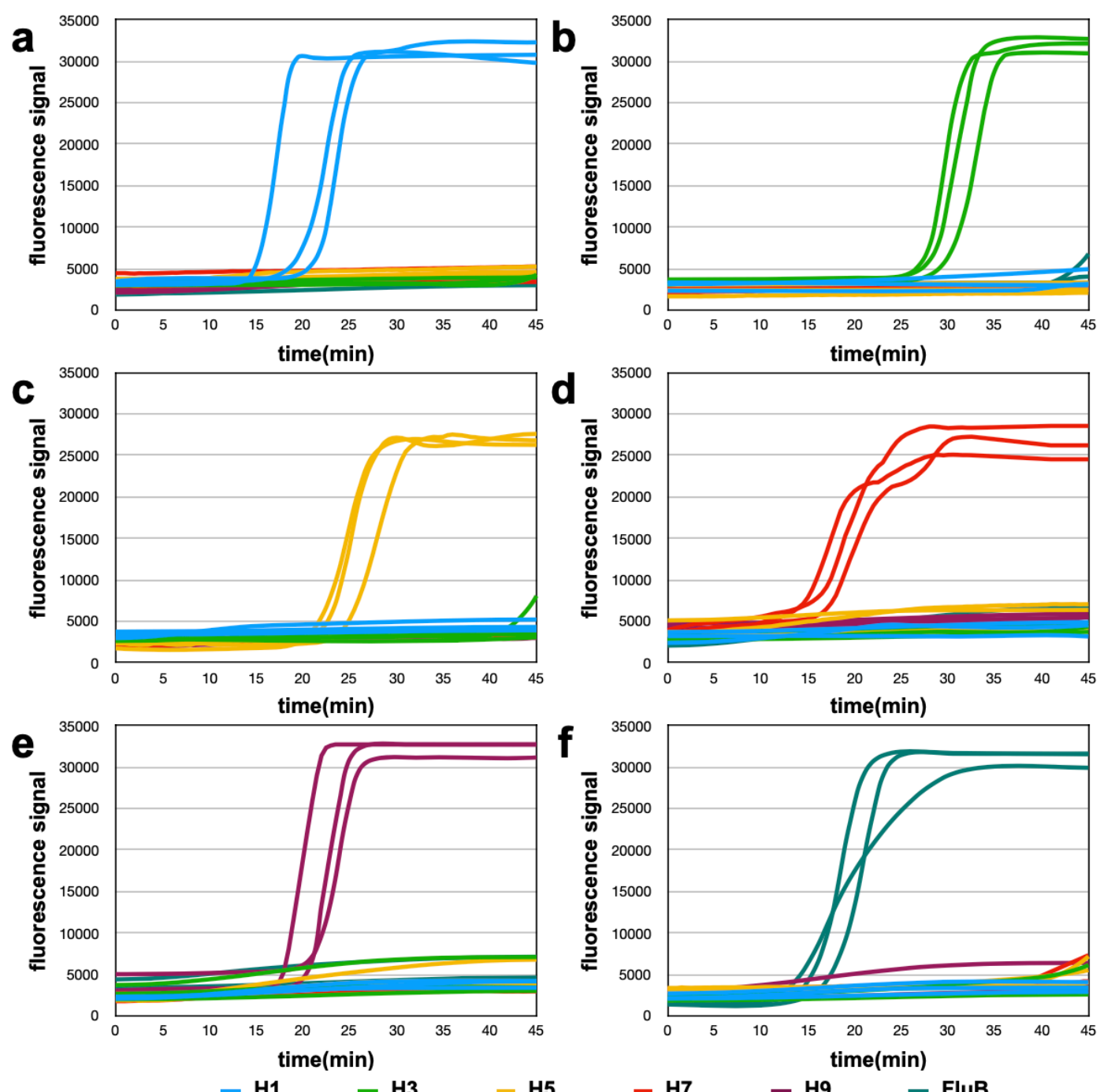

Figure S2: RT-LAMP results of clinical samples in the centrifugal tubes. a-f: Clinical samples RT-LAMP results for $\mathrm{H} 1, \mathrm{H} 3, \mathrm{H} 5, \mathrm{H} 7, \mathrm{H} 9$ and FluB, respectively. The blue, green, yellow, red, purple and cyan lines represent H1, H3, H5, H7, H9 and FluB, respectively. 